\title{
Certain mean-type fractional integral inequalities via different convexities with applications
}

\author{
Muhammad Samraiz ${ }^{1}$, Fakhra Nawaz' ${ }^{1}$ Sajid lqbal' ${ }^{2}$ Thabet Abdeljawad ${ }^{3,4,5^{*}}$ (D), Gauhar Rahman ${ }^{6}$ and \\ Kottakkaran Sooppy Nisar ${ }^{7}$
}

"Correspondence:

tabdeljawad@psu.edu.sa

${ }^{3}$ Department of Mathematics and

General Sciences, Prince Sultan

University, 11586 Riyadh, Saudi

Arabia

${ }^{4}$ Department of Medical Research,

China Medical University, 40402

Taichung, Taiwan

Full list of author information is

available at the end of the article

\section{Springer}

\begin{abstract}
In this paper, we establish certain generalized fractional integral inequalities of mean and trapezoid type for $(s+1)$-convex functions involving the $(k, s)$-Riemann-Liouville integrals. Moreover, we develop such integral inequalities for $h$-convex functions involving the $k$-conformable fractional integrals. The legitimacy of the derived results is demonstrated by plotting graphs. As applications of the derived inequalities, we obtain the classical Hermite-Hadamard and trapezoid inequalities.
\end{abstract}

MSC: Primary 26D15; secondary 26A33; 26A51; 26D07; 26D10

Keywords: Mean-type inequalities; $h$-convex; $(s+1)$-convex; $k$-conformable integral; $(k, s)$-Riemann-Liouville fractional integral; Conformable integral; Trapezoid inequalities

\section{Introduction}

The well known Hermite-Hadamard inequality for a convex function $\Psi: U \rightarrow \mathbb{R}$ on an interval $U$ of real numbers, with $\phi, \varphi \in U$ and $\phi<\varphi$ is given by

$$
\Psi\left(\frac{\phi+\varphi}{2}\right) \leq \frac{1}{\varphi-\phi} \int_{\phi}^{\varphi} \Psi(\xi) d \xi \leq \frac{\Psi(\phi)+\Psi(\varphi)}{2} .
$$

Numerous scientists examined this inequality and published various generalizations and extensions by using fractional integrals and derivatives $[5,8,15,16,18,19,23,25-$ $29,32,33]$. The theories of $k$ - and $(k, s)$-fractional operators are the more generalized way to express fractional calculus operators (see $[21,22,24])$. The classical fractional operators become special cases of such theories. Considering late developments in the theory of differential and integral equations, it is getting very hard to ignore the existence of integral inequalities that help determine the bounds on unknown functions. Applications of integral inequalities are important in various fields of science, like mathematics, physics, engineering, among others, we especially notice initial-value problems, the stability of linear transformation, integral differential equations, and impulse equations. We refer the readers $[1,3,4,6,7,12,13,20]$ for such applications in several branches of mathematics and the

(c) The Author(s) 2020. This article is licensed under a Creative Commons Attribution 4.0 International License, which permits use, sharing, adaptation, distribution and reproduction in any medium or format, as long as you give appropriate credit to the original author(s) and the source, provide a link to the Creative Commons licence, and indicate if changes were made. The images or other third party material in this article are included in the article's Creative Commons licence, unless indicated otherwise in a credit line to the material. If material is not included in the article's Creative Commons licence and your intended use is not permitted by statutory regulation or exceeds the permitted use, you will need to obtain permission directly from the copyright holder. To view a copy of this licence, visit http://creativecommons.org/licenses/by/4.0/. 
references therein. Firstly, we give some key definitions and mathematical fundamentals of the theory of fractional calculus which are utilized in this paper.

Definition 1.1 ([14]) A function $\psi:[\phi, \varphi] \rightarrow \mathbb{R}$ is called convex if the following inequality holds on an interval $[\phi, \varphi] \subseteq \mathbb{R}$ :

$$
\psi(v l+(1-v) r) \leq v \psi(l)+(1-v) \psi(r)
$$

where $l, r \in[\phi, \varphi]$, and $v \in[0,1]$.

Definition $1.2([24])$ Let $\psi$ be a continuous function on a finite interval $[\phi, \varphi]$. Then the left and right $(k, s)$-Riemann-Liouville fractional integrals of order $\chi>0$ are defined by

$$
F_{\phi^{+}, k}^{\chi, s} \psi(\tau)=\frac{(s+1)^{1-\frac{\chi}{k}}}{k \Gamma_{k}(\chi)} \int_{\phi}^{\tau}\left(\tau^{s+1}-\wp^{s+1}\right)^{\frac{\chi}{k}-1} \wp^{s} \psi(\wp) d \wp
$$

and

$$
F_{\varphi^{-,}, k}^{\chi, s} \psi(\tau)=\frac{(s+1)^{1-\frac{\chi}{k}}}{k \Gamma_{k}(\chi)} \int_{\tau}^{\varphi}\left(\wp^{s+1}-\tau^{s+1}\right)^{\frac{\chi}{k}-1} \wp^{s} \psi(\wp) d \wp
$$

respectively, where $k>0, s \in \mathbb{R} \backslash\{-1\}$.

Definition 1.3 ([10]) The left and right conformable fractional integral operators $\mathfrak{J}_{\phi^{+}}^{\chi, \beta}$ and $\mathfrak{J}_{\varphi^{-}}^{\chi, \beta}$ of order $\beta \in \mathbb{C}$, such that $\mathfrak{R e}(\beta)>0$ and $0<\chi \leq 1$, for $\psi \in L_{1}[\phi, \varphi]$ are defined by

$$
\mathfrak{J}_{\phi^{+}}^{\chi, \beta} \psi(\wp)=\frac{1}{\Gamma(\beta)} \int_{\phi}^{\wp}\left(\frac{(\wp-\phi)^{\chi}-(v-\phi)^{\chi}}{\chi}\right)^{\beta-1}(v-\phi)^{\chi-1} \psi(v) d v
$$

and

$$
\mathfrak{J}_{\varphi^{-}}^{\chi, \beta} \psi(\wp)=\frac{1}{\Gamma(\beta)} \int_{\wp}^{\varphi}\left(\frac{(\varphi-\wp)^{\chi}-(\varphi-v)^{\chi}}{\chi}\right)^{\beta-1}(\varphi-v)^{\chi-1} \psi(\nu) d \nu
$$

respectively, where $\Gamma$ is the Euler gamma function.

Definition 1.4 ([21]) The generalized left and right $k$-conformable fractional integral operators $\mathfrak{J}_{\phi^{+}, k}^{\chi, \beta}$ and $\mathfrak{J}_{\varphi^{-}, k}^{\chi, \beta}$ of order $\beta \in \mathbb{C}, \mathfrak{R e}(\beta)>0, k>0$ and $0<\chi \leq 1$, for $\psi \in L_{1, \chi}[\phi, \varphi]$ are defined by

$$
\mathfrak{J}_{\phi^{+}, k}^{\chi, \beta} \psi(\wp)=\frac{1}{k \Gamma_{k}(\beta)} \int_{\phi}^{\wp}\left(\frac{(\wp-\phi)^{\frac{\chi}{k}}-(v-\phi)^{\frac{\chi}{k}}}{\chi}\right)^{\frac{\beta}{k}-1}(v-\phi)^{\frac{\chi}{k}-1} \psi(\nu) d \nu
$$

and

$$
\mathfrak{J}_{\varphi^{-}, k}^{\chi, \beta} \psi(\wp)=\frac{1}{k \Gamma_{k}(\beta)} \int_{\wp}^{\varphi}\left(\frac{(\varphi-\wp)^{\frac{\chi}{k}}-(\varphi-v)^{\frac{\chi}{k}}}{\chi}\right)^{\frac{\beta}{k}-1}(\varphi-\nu)^{\frac{\chi}{k}-1} \psi(\nu) d \nu,
$$

respectively. 
Definition 1.5 ([11]) A function $\psi: D \subset \mathbb{R}^{+} \rightarrow \mathbb{R}^{+}$is called geometric-arithmetically $s$ convex on $D$ if $s \in(0,1], \forall \phi, \varphi \in D$ and $v \in[0,1]$, we have

$$
\psi\left(\phi^{v} \varphi^{1-\nu}\right) \leq v^{s} \psi(\phi)+(1-v)^{s} \psi(\varphi)
$$

We use the following notations in our upcoming results:

$S X(h, D)$-class of $h$-convex functions;

$S V(h, D)$-class of $h$-concave functions;

$K_{s}^{2}-s$-convex functions in the second sense;

$P(I)$-quasiconvex functions.

Sanja Varošanec presented the class of convex functions in [31] as follows:

Definition 1.6 Let $h: D \subset \mathbb{R} \rightarrow \mathbb{R}$ be a positive function. We say that $\psi: D \subset \mathbb{R} \rightarrow \mathbb{R}$ is $h$-convex, if $\psi$ is nonnegative and $\forall \phi, \varphi \in D, v \in(0,1)$, we have

$$
\psi(v \phi+(1-v) \varphi) \leq h(v) \psi(\phi)+h(1-v) \psi(\varphi) .
$$

If the inequality in (1.2) is reversed, then $\psi$ will be $h$-concave, i.e., $\psi \in S V(h, D)$. If $h(v)=v$, then all nonnegative convex functions belong to $S X(h, D)$ and all nonnegative concave functions belong to $S V(h, D)$; if $h(v)=1$, then $S X(h, D) \supseteq P(D)$ and if $h(v)=v^{s}$, where $s \in(0,1)$, then $S X(h, D) \supseteq K_{s}^{2}$.

The formal definition of the beta function given in [2] is stated as follows:

Definition 1.7 The classical beta function, also called the Euler integral of the first kind, is a special function defined by

$$
\mathbf{B}(x, y)=\int_{0}^{1} t^{x-1}(1-t)^{y-1} d t, \quad \mathfrak{R}(x)>0, \mathfrak{R}(y)>0 .
$$

Definition 1.8 ([17]) The integral form of the hypergeometric function is given as

$$
{ }_{2} \mathfrak{F}_{1}(u, v ; c ; z)=\frac{1}{\mathbf{B}(v, c-v)} \int_{0}^{1} \wp^{v-1}(1-\wp)^{c-v-1}(1-z \wp)^{-u} d \wp
$$

for $|z|<1, \mathbb{R}(c)>\mathbb{R}(v)>0$.

Definition 1.9 ([9]) Consider an interval $J \subset(0, \infty)=\mathbb{R}+$ and $(s+1) \in \mathbb{R} \backslash\{0\}$. A function $\psi: J \rightarrow \mathbb{R}$ is called $(s+1)$-convex if

$$
\psi\left(\left[\wp \phi^{s+1}+(1-\wp) \varphi^{s+1}\right]^{\frac{1}{s+1}}\right) \leq \wp \psi(\phi)+(1-\wp) \psi(\varphi),
$$

for all $\phi, \varphi \in J$ and $\wp \in[0,1]$. 
Lemma 1.10 ([30]) Let $\psi:[\phi, \varphi] \rightarrow \mathbb{R}$ be a differentiable function on $(\phi, \varphi)$ with $\phi<\varphi$ and $\psi \in L_{1}[\phi, \varphi]$. Then the following equality for fractional conformable integrals holds:

$$
\begin{aligned}
& \frac{\psi(\phi)+\psi(\varphi)}{2}-\frac{\chi^{\beta} \Gamma(\beta+1)}{2(\varphi-\phi)^{\chi \beta}}\left[\mathfrak{J}_{\phi^{+}}^{\chi, \beta} \psi(\varphi)+\mathfrak{J}_{\varphi^{-}}^{\chi, \beta} \psi(\phi)\right] \\
& =\frac{(\varphi-\phi)}{2} \int_{0}^{1}\left[\left(1-\wp^{\chi}\right)^{\beta}-\left(1-(1-\wp)^{\chi}\right)^{\beta}\right] \psi^{\prime}(\wp \phi+(1-\wp) \varphi) d \wp .
\end{aligned}
$$

\section{Inequalities involving $(k, s)$-Riemann-Liouville fractional integral}

This section includes mean-type inequalities for $(k, s)$-Riemann-Liouville fractional integral operators of order $\chi>0$.

Theorem 2.1 Let $\psi:[\phi, \varphi] \subset(0, \infty) \rightarrow \mathbb{R}$ be an $(s+1)$-convex function such that $\psi \in$ $L[\phi, \varphi]$. Then

(i) for $s>-1$, we have

$$
\begin{aligned}
\psi\left(\left[\frac{\phi^{s+1}+\varphi^{s+1}}{2}\right]^{\frac{1}{s+1}}\right) & \leq \frac{(s+1)^{\frac{\chi}{k}} \Gamma_{k}(\chi+k)}{2\left(\varphi^{s+1}-\phi^{s+1}\right)^{\frac{\chi}{k}}}\left[F_{\phi^{+}, k}^{\chi, s} \psi(\varphi)+F_{\varphi^{-}, k}^{\chi, s} \psi(\phi)\right] \\
& \leq \frac{\psi(\phi)+\psi(\varphi)}{2}
\end{aligned}
$$

and

(ii) for $s<-1$, we have

$$
\begin{aligned}
\psi\left(\left[\frac{\phi^{s+1}+\varphi^{s+1}}{2}\right]^{\frac{1}{s+1}}\right) & \leq \frac{(s+1)^{\frac{\chi}{k}} \Gamma_{k}(\chi+k)}{2\left(\phi^{s+1}-\varphi^{s+1}\right)^{\frac{\chi}{k}}}\left[F_{\phi^{+}, k}^{\chi, s} \psi(\varphi)+F_{\varphi^{-}, k}^{\chi, s} \psi(\phi)\right] \\
& \leq \frac{\psi(\phi)+\psi(\varphi)}{2} .
\end{aligned}
$$

Proof (i) Since $\psi$ is an $(s+1)$-convex function, we can write

$$
\begin{aligned}
& \psi\left(\left[v x^{s+1}+(1-v) y^{s+1}\right]^{\frac{1}{s+1}}\right) \leq v \psi(x)+(1-v) \psi(y), \\
& \psi\left(\left[(1-v) x^{s+1}+v y^{s+1}\right]^{\frac{1}{s+1}}\right) \leq(1-v) \psi(x)+v \psi(y) .
\end{aligned}
$$

Let $v=\frac{1}{2}$, then

$$
\psi\left(\left[\frac{x^{s+1}+y^{s+1}}{2}\right]^{\frac{1}{s+1}}\right) \leq \frac{\psi(x)+\psi(y)}{2} .
$$

Assume $x^{s+1}=v \phi^{s+1}+(1-v) \varphi^{s+1}$ and $y^{s+1}=(1-v) \phi^{s+1}+\nu \varphi^{s+1}$, then we have

$$
\begin{aligned}
& \psi\left(\left[\frac{\phi^{s+1}+\varphi^{s+1}}{2}\right]^{\frac{1}{s+1}}\right) \\
& \quad \leq \frac{1}{2}\left[\psi\left(\left[v \phi^{s+1}+(1-v) \varphi^{s+1}\right]^{\frac{1}{s+1}}\right)+\psi\left(\left[\phi^{s+1}(1-v)+\nu \varphi^{s+1}\right]^{\frac{1}{s+1}}\right)\right] .
\end{aligned}
$$


Multiplying the above inequality by $v^{\frac{x}{k}-1}$ and then integrating with respect to $v$ over $[0,1]$, we get

$$
\begin{aligned}
\frac{k}{\chi} \psi & \left(\left[\frac{\phi^{s+1}+\varphi^{s+1}}{2}\right]^{\frac{1}{s+1}}\right) \\
\leq & \frac{1}{2}\left[\int_{0}^{1} v^{\frac{\chi}{k}-1}\left[\psi\left(\left[v \phi^{s+1}+(1-v) \varphi^{s+1}\right]^{\frac{1}{s+1}}\right)\right] d v\right. \\
& \left.+\int_{0}^{1} v^{\frac{\chi}{k}-1}\left[\psi\left(\left[\phi^{s+1}(1-v)+v \varphi^{s+1}\right]^{\frac{1}{s+1}}\right)\right] d v\right] .
\end{aligned}
$$

Now, let $v \phi^{s+1}+(1-v) \varphi^{s+1}=u^{s+1}$ and $(1-v) \phi^{s+1}+v \varphi^{s+1}=v^{s+1}$, then (2.4) becomes

$$
\psi\left(\left[\frac{\phi^{s+1}+\varphi^{s+1}}{2}\right]^{\frac{1}{s+1}}\right) \leq \frac{(s+1)^{\frac{\chi}{k}} \Gamma_{k}(\chi+k)}{2\left(\varphi^{s+1}-\phi^{s+1}\right)^{\frac{\chi}{k}}}\left[F_{\phi^{+}, k}^{\chi, s} \psi(\varphi)+F_{\varphi^{-}, k}^{\chi, s} \psi(\phi)\right] .
$$

Now, by replacing $x=\phi, y=\varphi$ in (2.2) and (2.3), respectively, then adding, we have

$$
\psi\left(\left[v \phi^{s+1}+(1-v) \varphi^{s+1}\right]^{\frac{1}{s+1}}\right)+\psi\left(\left[(1-v) \phi^{s+1}+v \varphi^{s+1}\right]^{\frac{1}{s+1}}\right) \leq[\psi(\phi)+\psi(\varphi)] .
$$

Multiplying (2.6) by $v^{\frac{x}{k}-1}$ and then integrating over [0,1], we have

$$
\frac{(s+1)^{\frac{\chi}{k}} \Gamma_{k}(\chi+k)}{2\left(\varphi^{s+1}-\phi^{s+1}\right)^{\frac{\chi}{k}}}\left[F_{\phi^{+}, k}^{\chi, s} \psi(\varphi)+F_{\varphi^{-}, k}^{\chi, s} \psi(\phi)\right] \leq \frac{\psi(\phi)+\psi(\varphi)}{2} .
$$

Now, from (2.5) and (2.7), we get the desired result. This completes the proof of (i).

(ii) The proof of (ii) is similar to (i), so is omitted. Thus, the proof of the theorem is completed.

Example 2.2 By plotting the graphs of (2.1) for a convex function $\psi(\wp)=e^{\wp}$, we check that both inequalities are valid. It is known that the $(k, s)$-Riemann-Liouville fractional integrals of this function for $s=0$ are given by

$$
F_{\phi^{+}, k}^{\chi, 0} e^{\varphi}=\frac{1}{k \Gamma_{k}(\chi)} \int_{\phi}^{\varphi}(\varphi-\wp)^{\frac{\chi}{k}-1} e^{\wp} d \wp
$$

and

$$
F_{\varphi^{-}, k}^{\chi, 0} e^{\phi}=\frac{1}{k \Gamma_{k}(\chi)} \int_{\phi}^{\varphi}(\wp-\phi)^{\frac{\chi}{k}-1} e^{\wp} d \wp .
$$

By utilizing these expressions in the double inequality (2.1), we get

$$
2 e^{\frac{\phi+\varphi}{2}} \leq \frac{\chi}{k(\varphi-\phi)^{\frac{\chi}{k}}} \int_{0}^{1}\left[(\varphi-\wp)^{\frac{\chi}{k}-1}+(\wp-\phi)^{\frac{\chi}{k}-1}\right] e^{\wp} d \wp \leq e^{\phi}+e^{\varphi} .
$$

The three functions given by the left, middle, and right sides of the double inequality (2.10) are plotted in Fig. 1 against $\chi \in(0,1]$. The graphs of the functions show the validity of dual inequality. 
Samraiz et al. Journal of Inequalities and Applications

(2020) $2020: 208$

Page 6 of 19

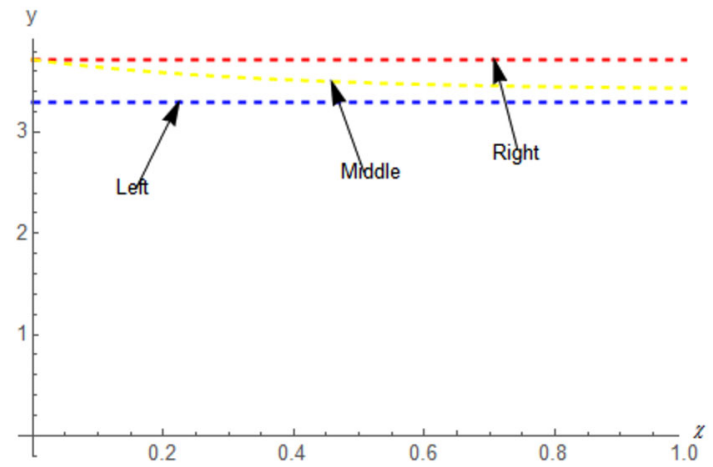

Figure 1 The graphs illustrate the validity of the double inequality (2.10) for the case $\phi=0, \varphi=1$, and $k=1$

Theorem 2.3 Let $\psi:[\phi, \varphi] \subset(0, \infty) \rightarrow \mathbb{R}$ be a differentiable function on $(\phi, \varphi)$ with $\phi<\varphi$, $s \in \mathbb{R} \backslash\{-1\}$ such that $\psi^{\prime} \in L[\phi, \varphi]$, then

(i) for $s>-1$, we have

$$
\begin{aligned}
& \frac{\psi(\phi)+\psi(\varphi)}{2}-\frac{(s+1)^{\frac{\chi}{k}} \Gamma_{k}(\chi+k)}{2\left(\varphi^{s+1}-\phi^{s+1}\right)^{\frac{\chi}{k}}}\left[F_{\phi^{+}, k}^{\chi, s} \psi(\varphi)+F_{\varphi^{-}, k}^{\chi, s} \psi(\phi)\right] \\
& =\frac{\left(\varphi^{s+1}-\phi^{s+1}\right)}{2(s+1)} \\
& \quad \times \int_{0}^{1}\left[(1-\wp)^{\frac{\chi}{k}}-(\wp)^{\frac{\chi}{k}}\right] N^{\frac{1}{s+1}-1} \psi^{\prime}\left[\left(\wp \phi^{s+1}+(1-\wp) \varphi^{s+1}\right)^{\frac{1}{s+1}}\right] d \wp,
\end{aligned}
$$

where $N=\left(\wp \phi^{s+1}+(1-\wp) \varphi^{s+1}\right)$;

(ii) for $s<-1$, we can write

$$
\begin{aligned}
& \frac{\psi(\phi)+\psi(\varphi)}{2}-\frac{(s+1)^{\frac{\chi}{k}} \Gamma_{k}(\chi+k)}{2\left(\phi^{s+1}-\varphi^{s+1}\right)^{\frac{\chi}{k}}}\left[F_{\phi^{+}, k}^{\chi, s} \psi(\varphi)+F_{\varphi^{-}, k}^{\chi, s} \psi(\phi)\right] \\
& =\frac{\left(\phi^{s+1}-\varphi^{s+1}\right)}{2(s+1)} \\
& \quad \times \int_{0}^{1}\left[(1-\wp)^{\frac{\chi}{k}}-(\wp)^{\frac{\chi}{k}}\right] M^{\frac{1}{s+1}-1} \psi^{\prime}\left[\left(\wp \varphi^{s+1}+(1-\wp) \phi^{s+1}\right)^{\frac{1}{s+1}}\right] d \wp,
\end{aligned}
$$

where $M=\left(\wp \varphi^{s+1}+(1-\wp) \phi^{s+1}\right)$.

Proof (i) Consider

$$
\begin{aligned}
I= & \int_{0}^{1}\left[(1-\wp)^{\frac{\chi}{k}}-(\wp)^{\frac{\chi}{k}}\right] N^{\frac{1}{s+1}-1} \psi^{\prime}\left[\left(\wp \phi^{s+1}+(1-\wp) \varphi^{s+1}\right)^{\frac{1}{s+1}}\right] d \wp \\
= & \int_{0}^{1}(1-\wp)^{\frac{\chi}{k}} N^{\frac{1}{s+1}-1} \psi^{\prime}\left[\left(\wp \phi^{s+1}+(1-\wp) \varphi^{s+1}\right)^{\frac{1}{s+1}}\right] d \wp \\
& -\int_{0}^{1} \wp^{\frac{\chi}{k}} N^{\frac{1}{s+1}-1} \psi^{\prime}\left[\left(\wp \phi^{s+1}+(1-\wp) \varphi^{s+1}\right)^{\frac{1}{s+1}}\right] d \wp \\
= & I_{1}-I_{2} .
\end{aligned}
$$


Integrating $I_{1}$ by parts, we get

$$
\begin{aligned}
I_{1}= & \frac{s+1}{\varphi^{s+1}-\phi^{s+1}} \psi(\varphi) \\
& -\frac{\chi(s+1)}{k\left(\varphi^{s+1}-\phi^{s+1}\right)} \int_{0}^{1}(1-\wp)^{\frac{\chi}{k}-1} \psi\left(\left(\wp \phi^{s+1}+(1-\wp) \varphi^{s+1}\right)^{\frac{1}{s+1}}\right) d \wp .
\end{aligned}
$$

Let $\wp \phi^{s+1}+(1-\wp) \varphi^{s+1}=u^{s+1}$, then $(2.12)$ becomes

$$
\begin{aligned}
I_{1} & =\frac{s+1}{\varphi^{s+1}-\phi^{s+1}} \psi(\varphi)-\frac{\chi(s+1)^{2}}{k\left(\varphi^{s+1}-\phi^{s+1}\right)^{\frac{\chi}{k}+1}} \int_{\phi}^{\varphi}\left(u^{s+1}-\phi^{s+1}\right)^{\frac{\chi}{k}-1} u^{s} \psi(u) d u \\
& =\frac{s+1}{\varphi^{s+1}-\phi^{s+1}} \psi(\varphi)-\frac{(s+1)^{\frac{\chi}{k}+1} \Gamma_{k}(\chi+k)}{\left(\varphi^{s+1}-\phi^{s+1}\right)^{\frac{\chi}{k}+1}} F_{\varphi^{-}, k}^{\chi, s} \psi(\phi) .
\end{aligned}
$$

Now, integrating $I_{2}$ by parts, we have the equation

$$
\begin{aligned}
I_{2}= & -\frac{s+1}{\varphi^{s+1}-\phi^{s+1}} \psi(\phi) \\
& +\frac{\chi(s+1)}{k\left(\varphi^{s+1}-\phi^{s+1}\right)} \int_{0}^{1} \wp^{\frac{\chi}{k}-1} \psi\left(\left(\wp \phi^{s+1}+(1-\wp) \varphi^{s+1}\right)^{\frac{1}{s+1}}\right) d \wp .
\end{aligned}
$$

Let $\wp \phi^{s+1}+(1-\wp) \varphi^{s+1}=u^{s+1}$, then (2.13) becomes

$$
\begin{aligned}
I_{2} & =-\frac{s+1}{\varphi^{s+1}-\phi^{s+1}} \psi(\phi)+\frac{\chi(s+1)^{2}}{k\left(\varphi^{s+1}-\phi^{s+1}\right)^{\frac{\chi}{k}+1}} \int_{\phi}^{\varphi}\left(\varphi^{s+1}-u^{s+1}\right)^{\frac{\chi}{k}-1} u^{s} \psi(u) d u \\
& =-\frac{s+1}{\varphi^{s+1}-\phi^{s+1}} \psi(\phi)+\frac{(s+1)^{\frac{\chi}{k}+1} \Gamma_{k}(\chi+k)}{\left(\varphi^{s+1}-\phi^{s+1}\right)^{\frac{\chi}{k}+1}} F_{\phi^{+}, k}^{\chi, s} \psi(\varphi) .
\end{aligned}
$$

Now, by using the values of $I_{1}$ and $I_{2}$ in (2.11), we get

$$
\begin{aligned}
I= & \frac{s+1}{\varphi^{s+1}-\phi^{s+1}} \psi(\varphi)-\frac{(s+1)^{\frac{\chi}{k}+1} \Gamma_{k}(\chi+k)}{\left(\varphi^{s+1}-\phi^{s+1}\right)^{\frac{\chi}{k}+1}} F_{\varphi^{-}, k}^{\chi, s} \psi(\phi) \\
& +\frac{s+1}{\varphi^{s+1}-\phi^{s+1}} \psi(\phi)-\frac{(s+1)^{\frac{\chi}{k}+1} \Gamma_{k}(\chi+k)}{\left(\varphi^{s+1}-\phi^{s+1}\right)^{\frac{\chi}{k}+1}} F_{\phi^{+}, k}^{\chi, s} \psi(\varphi) \\
= & \frac{(s+1)(\psi(\phi)+\psi(\varphi))}{\varphi^{s+1}-\phi^{s+1}}-\frac{(s+1)^{\frac{\chi}{k}+1} \Gamma_{k}(\chi+k)}{\left(\varphi^{s+1}-\phi^{s+1}\right)^{\frac{\chi}{k}+1}}\left[F_{\phi^{+}, k}^{\chi, s} \psi(\varphi)+F_{\varphi^{-}, k}^{\chi, s} \psi(\phi)\right],
\end{aligned}
$$

which gives

$$
\begin{aligned}
& \frac{\psi(\phi)+\psi(\varphi)}{2}-\frac{(s+1)^{\frac{\chi}{k}} \Gamma_{k}(\chi+k)}{2\left(\varphi^{s+1}-\phi^{s+1}\right)^{\frac{\chi}{k}}}\left[F_{\phi^{+}, k}^{\chi, s} \psi(\varphi)+F_{\varphi^{-}, k}^{\chi, s} \psi(\phi)\right] \\
& \quad=\frac{\left(\varphi^{s+1}-\phi^{s+1}\right)}{2(s+1)} \int_{0}^{1}\left[(1-\wp)^{\frac{\chi}{k}}-(\wp)^{\frac{\chi}{k}}\right] N^{\frac{1}{s+1}-1} \psi^{\prime}\left[\left(\wp \phi^{s+1}+(1-\wp) \varphi^{s+1}\right)^{\frac{1}{s+1}}\right] d \wp .
\end{aligned}
$$

This completes the proof of (i)

(ii) The proof of (ii) is similar to (i), so is omitted. Thus, the proof of the theorem is completed. 
Theorem 2.4 Let $\psi:[\phi, \varphi] \subset(0, \infty) \rightarrow \mathbb{R}$ be a differentiable function on $(\phi, \varphi), \phi<\varphi$, such that $\psi^{\prime} \in L[\phi, \varphi]$. If $\left|\psi^{\prime}\right|^{g}$, where $g>1$, is $(s+1)$-convex, then

(i) for $s>-1$,

$$
\begin{aligned}
& \left|\frac{\psi(\phi)+\psi(\varphi)}{2}-\frac{(s+1)^{\frac{\chi}{k}} \Gamma_{k}(\chi+k)}{2\left(\varphi^{s+1}-\phi^{s+1}\right)^{\frac{\chi}{k}}}\left[F_{\phi^{+}, k}^{\chi, s} \psi(\varphi)+F_{\varphi^{-}, k}^{\chi, s} \psi(\phi)\right]\right| \\
& \leq \frac{\left(\varphi^{s+1}-\phi^{s+1}\right)}{2(s+1)}\left\{\varphi^{-s}{ }_{2} \mathfrak{F}_{1}\left(\frac{s}{s+1}, 1 ; 2 ; 1-\frac{\phi^{s+1}}{\varphi^{s+1}}\right)\right\}^{1-\frac{1}{g}}\left[\left(\frac{1}{\frac{\chi}{k}+1}\right)\right. \\
& \left.\quad \times\left[\left|\psi^{\prime}(\phi)\right|^{g}+\left|\psi^{\prime}(\varphi)\right|^{g}\right]\right]^{\frac{1}{g}},
\end{aligned}
$$

(ii) for $s<-1$,

$$
\begin{aligned}
& \left|\frac{\psi(\phi)+\psi(\varphi)}{2}-\frac{(s+1)^{\frac{\chi}{k}} \Gamma_{k}(\chi+k)}{2\left(\varphi^{s+1}-\phi^{s+1}\right)^{\frac{\chi}{k}}}\left[F_{\phi^{+}, k}^{\chi, s} \psi(\varphi)+F_{\varphi^{-}, k}^{\chi, s} \psi(\phi)\right]\right| \\
& \leq \frac{\left(\phi^{s+1}-\varphi^{s+1}\right)}{2(s+1)}\left\{\phi^{-s}{ }_{2} \mathfrak{F}_{1}\left(\frac{s}{s+1}, 1 ; 2 ; 1-\frac{\varphi^{s+1}}{\phi^{s+1}}\right)\right\}^{1-\frac{1}{g}}\left[\left(\frac{1}{\frac{\chi}{k}+1}\right)\right. \\
& \left.\quad \times\left[\left|\psi^{\prime}(\phi)\right|^{g}+\left|\psi^{\prime}(\varphi)\right|^{g}\right]\right]^{\frac{1}{g}} .
\end{aligned}
$$

Proof (i) Applying Theorem 2.3, modulus property, Hölder's inequality, and $(s+1)$ convexity of $\left|\psi^{\prime}\right|^{g}$, we get

$$
\begin{aligned}
&\left|\frac{\psi(\phi)+\psi(\varphi)}{2}-\frac{(s+1)^{\frac{\chi}{k}} \Gamma_{k}(\chi+k)}{2\left(\varphi^{s+1}-\phi^{s+1}\right)^{\frac{\chi}{k}}}\left[F_{\phi^{+}, k}^{\chi, s} \psi(\varphi)+F_{\varphi^{-}, k}^{\chi, s} \psi(\phi)\right]\right| \\
&=\left|\frac{\left(\varphi^{s+1}-\phi^{s+1}\right)}{2(s+1)} \int_{0}^{1}\left[(1-\wp)^{\frac{\chi}{k}}-(\wp)^{\frac{\chi}{k}}\right] N^{\frac{1}{s+1}-1} \psi^{\prime}\left[\left(\wp \phi^{s+1}+(1-\wp) \varphi^{s+1}\right)^{\frac{1}{s+1}}\right] d \wp\right| \\
& \leq\left|\frac{\left(\varphi^{s+1}-\phi^{s+1}\right)}{2(s+1)} \int_{0}^{1}\left[(1-\wp)^{\frac{\chi}{k}}+(\wp)^{\frac{\chi}{k}}\right] N^{\frac{1}{s+1}-1} \psi^{\prime}\left[\left(\wp \phi^{s+1}+(1-\wp) \varphi^{s+1}\right)^{\frac{1}{s+1}}\right] d \wp\right| \\
& \leq \frac{\left(\varphi^{s+1}-\phi^{s+1}\right)}{2(s+1)}\left(\int_{0}^{1} N^{\frac{1}{s+1}-1} d \wp\right)^{1-\frac{1}{g}} \\
& \times\left(\int_{0}^{1}\left[(1-\wp)^{\frac{\chi}{k}}+(\wp)^{\frac{\chi}{k}}\right] \mid \psi^{\prime}\left[\left.\left(\wp \phi^{s+1}+(1-\wp) \varphi^{s+1}\right)^{\frac{1}{s+1}}\right|^{g} d \wp\right)^{\frac{1}{g}}\right. \\
& \leq \frac{\left(\varphi^{s+1}-\phi^{s+1}\right)}{2(s+1)}\left(\int_{0}^{1} N^{\frac{1}{s+1}-1} d \wp\right)^{1-\frac{1}{g}} \\
& \times\left(\int_{0}^{1}\left[(1-\wp)^{\frac{\chi}{k}}+(\wp)^{\frac{\chi}{k}}\right]\left[\wp\left|\psi^{\prime}(\phi)\right|^{g}+(1-\wp) \mid \psi^{\prime}(\varphi)^{g}\right] d \wp\right)^{\frac{1}{g}} \\
&= \frac{\left(\varphi^{s+1}-\phi^{s+1}\right)}{2(s+1)}\left\{\varphi^{-s}{ }_{2} \mathfrak{F}_{1}\left(\frac{s}{s+1}, 1 ; 2 ; 1-\frac{\phi^{s+1}}{\varphi^{s+1}}\right)\right\}^{1-\frac{1}{g}}\left[\left(\frac{1}{\frac{\chi}{k}+1}\right)\right. \\
&\left.\times\left[\left|\psi^{\prime}(\phi)\right|^{g}+\left|\psi^{\prime}(\varphi)\right|^{g}\right]\right]^{\frac{1}{g}} .
\end{aligned}
$$

This completes the proof of (i). 


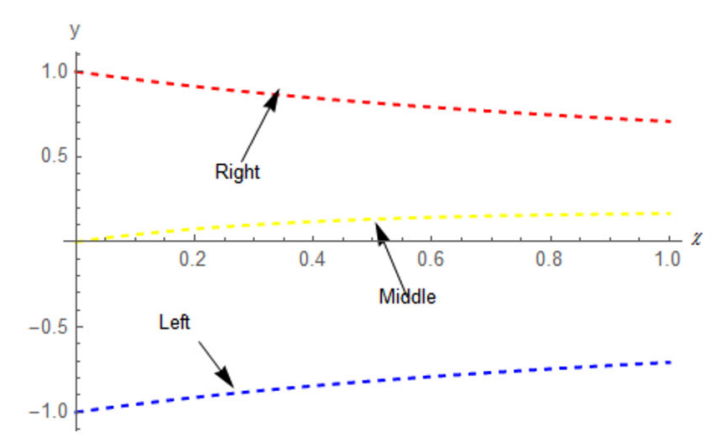

Figure 2 The graphs illustrate the result of the double inequality given by (2.15) for the case $\phi=0, \varphi=1$, and $k=1$

(ii) The proof of (ii) is similar to (i), so is omitted. Thus, the proof of the theorem is completed.

Example 2.5 By plotting the graphs of inequalities of Theorem 2.4 for the convex function $\psi(\wp)=\wp^{2}$ and $g=2$, we prove the validity of the results. Substitution of (2.8) and (2.9) into inequality (2.14) gives

$$
\begin{aligned}
& \left|\frac{\phi^{2}+\varphi^{2}}{2}-\frac{\chi}{2 k(\varphi-\phi)^{\frac{\chi}{k}}} \int_{0}^{1}\left[(\varphi-\wp)^{\frac{\chi}{k}-1}+(\wp-\phi)^{\frac{\chi}{k}-1}\right] x^{2} d \wp\right| \\
& \quad \leq(\varphi-\phi)\left(\frac{k}{\chi+k}\right)^{\frac{1}{2}}\left(\phi^{2}+\varphi^{2}\right)^{\frac{1}{2}}
\end{aligned}
$$

The three functions given by the left, middle and right sides of the double inequality (2.15) are plotted in Fig. 2 against $\chi \in(0,1]$. The graphs of the functions illustrate the validity of both inequalities.

\section{Inequalities involving conformable fractional integral operator}

This section contains mean-type inequalities for conformable fractional integral operators by using $h$-convexity.

Theorem 3.1 Let $\mathfrak{J}_{\phi^{+}}^{\chi, \beta}$ and $\mathfrak{J}_{\varphi^{-}}^{\chi, \beta}$ be the left-and right-sided generalized conformable fractional integrals operators of order $\beta \in \mathbb{C}, \mathfrak{R e}(\beta)>0$ and $0<\chi \leq 1$. Let $\psi:[\phi, \varphi] \rightarrow \mathbb{R}$ be a positive mapping with $\psi \in L_{1}[\phi, \varphi]$ and $0 \leq \phi<\varphi$. If $\psi$ is $h$-convex on $[\phi, \varphi]$, then

$$
\begin{aligned}
\psi\left(\frac{\phi+\varphi}{2}\right) \\
\leq \frac{h\left(\frac{1}{2}\right) \chi^{\beta} \Gamma(\beta+1)}{(\varphi-\phi)^{\chi \beta}}\left[\mathfrak{J}_{\phi^{+}}^{\chi, \beta} \psi(\varphi)+\mathfrak{J}_{\varphi^{-}}^{\chi, \beta} \psi(\phi)\right] \\
\leq \chi \beta h\left(\frac{1}{2}\right)[\psi(\phi)+\psi(\varphi)] \\
\quad \times \int_{0}^{1}\left(1-v^{\chi}\right)^{\beta-1} v^{\chi-1}[h(\nu)+h(1-v)] d \nu .
\end{aligned}
$$


Proof Since $\psi$ is $h$-convex function, we can write

$$
\psi(v x+(1-v) y) \leq h(v) \psi(x)+h(1-v) \psi(y)
$$

and

$$
\psi((1-v) x+v y) \leq h(1-v) \psi(x)+h(v) \psi(y) .
$$

Let $v=\frac{1}{2}$, then

$$
\psi\left(\frac{x+y}{2}\right) \leq h\left(\frac{1}{2}\right) \psi(x)+h\left(\frac{1}{2}\right) \psi(y)=h\left(\frac{1}{2}\right)[\psi(x)+\psi(y)]
$$

Assume $x=v \phi+(1-v) \varphi$ and $y=\phi(1-v)+\varphi v$, then we have

$$
\psi\left(\frac{\phi+\varphi}{2}\right) \leq h\left(\frac{1}{2}\right)[\psi(\nu \phi+(1-v) \varphi)+\psi(\phi(1-v)+\nu \varphi)]
$$

Multiplying the above inequality by $\left(1-v^{\chi}\right)^{\beta-1} v^{\chi-1}$ and then integrating with respect to $v$ over $[0,1]$, we get

$$
\begin{aligned}
\psi\left(\frac{\phi+\varphi}{2}\right) \int_{0}^{1}\left(1-v^{\chi}\right)^{\beta-1} v^{\chi-1} d t \\
\leq h\left(\frac{1}{2}\right)\left[\int_{0}^{1}\left(1-v^{\chi}\right)^{\beta-1} v^{\chi-1} \psi(v \phi+(1-v) \varphi) d v\right. \\
\left.\quad+\int_{0}^{1}\left(1-v^{\chi}\right)^{\beta-1} v^{\chi-1} \psi((1-v) \phi+v \varphi) d v\right],
\end{aligned}
$$

which can also be written as

$$
\begin{aligned}
\frac{1}{\chi \beta} \psi\left(\frac{\phi+\varphi}{2}\right) \\
\leq h\left(\frac{1}{2}\right)\left[\int_{0}^{1}\left(1-v^{\chi}\right)^{\beta-1} v^{\chi-1} \psi(v \phi+(1-v) \varphi) d v\right] \\
\quad+h\left(\frac{1}{2}\right)\left[\int_{0}^{1}\left(1-v^{\chi}\right)^{\beta-1} v^{\chi-1} \psi((1-v) \phi+v \varphi) d v\right] .
\end{aligned}
$$

Now, let $\nu \phi+(1-v) \varphi=u$ and $(1-v) \phi+v \varphi=v$, then (3.4) becomes

$$
\begin{aligned}
\frac{1}{\chi \beta} \psi & \left(\frac{\phi+\varphi}{2}\right) \\
\leq & \frac{h\left(\frac{1}{2}\right) \chi^{\beta-1} \Gamma(\beta)}{(\varphi-\phi)^{\chi \beta}}\left[\frac{\chi^{1-\beta}}{\Gamma(\beta)} \int_{\phi}^{\varphi}\left((\varphi-\phi)^{\chi}-(\varphi-u)^{\chi}\right)^{\beta-1}(\varphi-u)^{\chi-1} \psi(u) d u\right. \\
& \left.+\frac{\chi^{1-\beta}}{\Gamma(\beta)} \int_{\phi}^{\varphi}\left((\varphi-\phi)^{\chi}-(v-\phi)^{\chi}\right)^{\beta-1}(v-\phi)^{\chi-1} \psi(v) d v\right]
\end{aligned}
$$

which can be written as

$$
\psi\left(\frac{\phi+\varphi}{2}\right) \leq \frac{h\left(\frac{1}{2}\right) \chi^{\beta} \Gamma(\beta+1)}{(\varphi-\phi)^{\chi \beta}}\left[\mathfrak{J}_{\phi^{+}}^{\chi, \beta} \psi(\varphi)+\mathfrak{J}_{\varphi^{-}}^{\chi, \beta} \psi(\phi)\right] .
$$


Now, by replacing $x=\phi, y=\varphi$ in (3.2) and (3.3), respectively, then adding we have

$$
\psi(v \phi+(1-v) \varphi)+\psi((1-v) \phi+v \varphi) \leq[h(v)+h(1-v)][\psi(\phi)+\psi(\varphi)]
$$

Multiplying (3.6) by $\left(1-v^{\chi}\right)^{\beta-1} v^{\chi-1}$ and then integrating over $[0,1]$, we have

$$
\begin{aligned}
& \frac{h\left(\frac{1}{2}\right) \chi^{\beta} \Gamma(\beta+1)}{(\varphi-\phi)^{\chi \beta}}\left[\mathfrak{J}_{\phi^{+}}^{\chi, \beta} \psi(\varphi)+\mathfrak{J}_{\varphi^{-}}^{\chi, \beta} \psi(\phi)\right] \\
& \quad \leq \chi \beta h\left(\frac{1}{2}\right)[\psi(\phi)+\psi(\varphi)] \int_{0}^{1}\left(1-v^{\chi}\right)^{\beta-1} v^{\chi-1}[h(v)+h(1-v)] d \nu .
\end{aligned}
$$

Now, from (3.5) and (3.7), we get the desired result.

Example 3.2 We verify the result of Theorem 3.1 for the convex function $\psi(\wp)=e^{2 \wp}$ and $h(v)=v$. It is known that the conformable fractional integrals of this function for $\beta=1$ are given by

$$
\mathfrak{J}_{\phi^{+}}^{\chi, 1} \psi(\varphi)=\int_{\phi}^{\varphi}(v-\phi)^{\chi-1} e^{2 v} d v
$$

and

$$
\mathfrak{J}_{\varphi^{-}}^{\chi, 1} \psi(\phi)=\int_{\phi}^{\varphi}(\varphi-v)^{\chi-1} e^{2 v} d v
$$

Substituting these expressions into inequality (3.1), we get

$$
2 e^{(\phi+\varphi)} \leq \frac{\chi}{(\varphi-\phi)^{\chi}} \int_{0}^{1}\left[(\varphi-v)^{\chi-1}+(\nu-\phi)^{\chi-1}\right] e^{2 \nu} d \nu \leq\left[e^{2 \phi}+e^{2 \varphi}\right] .
$$

The three functions given by the left, middle, and right sides of this double inequality are plotted in Fig. 3 against $\chi \in(0,1]$ to show clearly that both inequalities are valid.

Theorem 3.3 Let $\psi:[\phi, \varphi] \rightarrow \mathbb{R}$ be a differentiable function on $(\phi, \varphi)$ with $\psi^{\prime} \in L_{1}[\phi, \varphi]$. If $\left|\psi^{\prime}\right|$ is an h-convex function on $[\phi, \varphi]$, then the following inequality for fractional con-

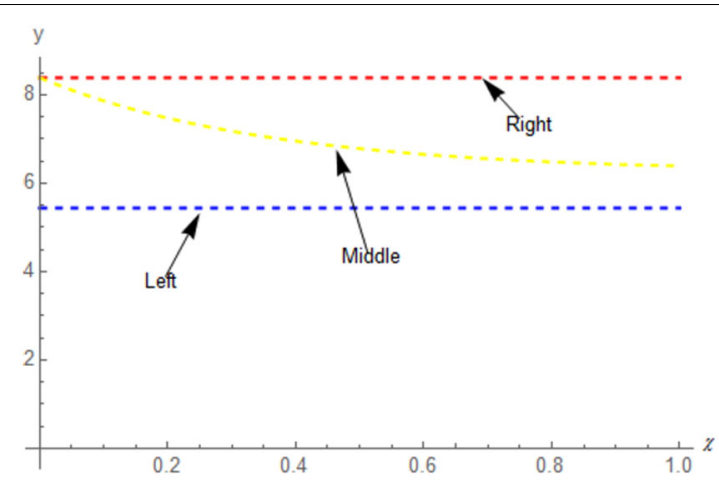

Figure 3 The graphs illustrate the validity of the dual inequality (3.10) for the case $\phi=0$ and $\varphi=1$ 
formable integrals holds:

$$
\begin{aligned}
& \left|\frac{\psi(\phi)+\psi(\varphi)}{2}-\frac{\chi^{\beta} \Gamma(\beta+1)}{2(\varphi-\phi)^{\chi \beta}}\left[\mathfrak{J}_{\phi^{+}}^{\chi, \beta} \psi(\varphi)+\mathfrak{J}_{\varphi^{-}}^{\chi, \beta} \psi(\phi)\right]\right| \\
& \leq \frac{\varphi-\phi}{2}\left[\left|\psi^{\prime}(\phi)\right| \int_{0}^{1}\left[\left(1-\wp^{\chi}\right)^{\beta}+\left(1-(1-\wp)^{\chi}\right)^{\beta}\right] h(\wp) d \wp\right. \\
& \left.\quad+\left|\psi^{\prime}(\varphi)\right| \int_{0}^{1}\left[\left(1-\wp^{\chi}\right)^{\beta}+\left(1-(1-\wp)^{\chi}\right)^{\beta}\right] h(1-\wp) d \wp\right] .
\end{aligned}
$$

Proof By using Lemma 1.10, modulus property, and $h$-convexity, we have

$$
\begin{aligned}
\mid & \frac{\psi(\phi)+\psi(\varphi)}{2}-\frac{\chi^{\beta} \Gamma(\beta+1)}{2(\varphi-\phi)^{\chi \beta}}\left[\mathfrak{J}_{\phi^{+}}^{\chi, \beta} \psi(\varphi)+\mathfrak{J}_{\varphi^{-}}^{\chi, \beta} \psi(\phi)\right] \mid \\
= & \left|\frac{(\varphi-\phi)}{2} \int_{0}^{1}\left[\left(1-\wp^{\chi}\right)^{\beta}-\left(1-(1-\wp)^{\chi}\right)^{\beta}\right] \psi^{\prime}(\wp \phi+(1-\wp) \varphi) d \wp\right| \\
\leq & \frac{(\varphi-\phi)}{2}\left|\int_{0}^{1}\left[\left(1-\wp^{\chi}\right)^{\beta}+\left(1-(1-\wp)^{\chi}\right)^{\beta}\right] \psi^{\prime}(\wp \phi+(1-\wp) \varphi) d \wp\right| \\
\leq & \frac{(\varphi-\phi)}{2} \int_{0}^{1}\left[\left(1-\wp^{\chi}\right)^{\beta}+\left(1-(1-\wp)^{\chi}\right)^{\beta}\right]\left[h(\wp)\left|\psi^{\prime}(\phi)\right|+h(1-\wp)\left|\psi^{\prime}(\varphi)\right|\right] d \wp \\
= & \frac{(\varphi-\phi)}{2}\left[\left|\psi^{\prime}(\phi)\right| \int_{0}^{1}\left[\left(1-\wp^{\chi}\right)^{\beta}+\left(1-(1-\wp)^{\chi}\right)^{\beta}\right] h(\wp) d \wp\right. \\
& \left.+\left|\psi^{\prime}(\varphi)\right| \int_{0}^{1}\left[\left(1-\wp^{\chi}\right)^{\beta}+\left(1-(1-\wp)^{\chi}\right)^{\beta}\right] h(1-\wp) d \wp\right],
\end{aligned}
$$

which completes the proof.

Example 3.4 We verify the result of Theorem 3.3 for the convex function $\psi(v)=e^{v}, \beta=1$, and $h(v)=v$. In this case, inequality (3.11) is given by

$$
-\frac{\chi(1+e)}{1+\chi} \leq(1+e)-\chi \int_{0}^{1}\left[(\nu-\phi)^{\chi-1}+(\varphi-v)^{\chi-1}\right] e^{v} d v \leq \frac{\chi(1+e)}{1+\chi} .
$$

The three functions given by the left, middle, and right sides of this double inequality are plotted in Fig. 4 against $\chi \in(0,1]$ to show that both inequalities are valid.

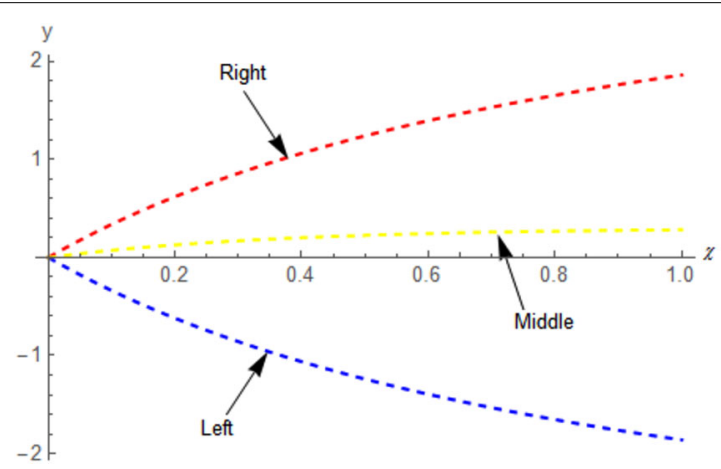

Figure 4 The graphs illustrate validity of the double inequality given by (3.12) for the case $\phi=0, \varphi=1$, and $0<\chi \leq 1$ 
Corollary 3.5 If we take $h(\wp)=1$ in Theorem 3.3 , then we get the result:

$$
\begin{aligned}
& \left|\frac{\psi(\phi)+\psi(\varphi)}{2}-\frac{\chi^{\beta} \Gamma(\beta+1)}{2(\varphi-\phi)^{\chi \beta}}\left[\mathfrak{J}_{\phi^{+}}^{\chi, \beta} \psi(\varphi)+\mathfrak{J}_{\varphi^{-}}^{\chi, \beta} \psi(\phi)\right]\right| \\
& \leq \frac{(\varphi-\phi)}{\chi}\left[\left|\psi^{\prime}(\phi)\right|+\left|\psi^{\prime}(\varphi)\right|\right] \mathbf{B}\left(\frac{1}{\chi}, \beta+1\right),
\end{aligned}
$$

where $\mathbf{B}$ denotes the usual beta function.

Corollary 3.6 If we take $h(\wp)=\wp$ in Theorem 3.3 , then we get the result for simple convex function presented below:

$$
\begin{aligned}
& \left|\frac{\psi(\phi)+\psi(\varphi)}{2}-\frac{\chi^{\beta} \Gamma(\beta+1)}{2(\varphi-\phi)^{\chi \beta}}\left[\mathfrak{J}_{\phi^{+}}^{\chi, \beta} \psi(\varphi)+\mathfrak{J}_{\varphi^{-}}^{\chi, \beta} \psi(\phi)\right]\right| \\
& \leq \frac{(\varphi-\phi)}{\chi}\left[\frac{\left|\psi^{\prime}(\phi)\right|+\left|\psi^{\prime}(\varphi)\right|}{2}\right] \mathbf{B}\left(\frac{1}{\chi}, \beta+1\right) .
\end{aligned}
$$

Corollary 3.7 If we take $h(\wp)=\wp^{s}$ in Theorem 3.3, where $s \in(0,1)$, then the result for geometric-arithmetically s-convex functions is as follows:

$$
\begin{aligned}
& \left|\frac{\psi(\phi)+\psi(\varphi)}{2}-\frac{\chi^{\beta} \Gamma(\beta+1)}{2(\varphi-\phi)^{\chi \beta}}\left[\mathfrak{J}_{\phi^{+}}^{\chi, \beta} \psi(\varphi)+\mathfrak{J}_{\varphi^{-}}^{\chi, \beta} \psi(\phi)\right]\right| \\
& \leq \frac{(\varphi-\phi)}{\chi}\left[\frac{\left|\psi^{\prime}(\phi)\right|+\left|\psi^{\prime}(\varphi)\right|}{2}\right]\left[\mathbf{B}\left(\frac{s+1}{\chi}, \beta+1\right)\right. \\
& \left.\quad+\mathbf{B}\left(\frac{1}{\chi}, \beta+1\right) \mathfrak{F}_{1}\left(-s, \frac{1}{\chi} ; \frac{\chi+\chi \beta+1}{\chi} ; u^{\frac{1}{\chi}-1}\right)\right] .
\end{aligned}
$$

\section{Inequalities involving generalized $k$-conformable fractional integral} operators

In this section, mean-type inequalities for $k$-conformable fractional integral operator by using $h$-convexity are established.

Theorem 4.1 Let $\mathfrak{J}_{\phi^{+}, k}^{\chi, \beta}$ and $\mathfrak{J}_{\varphi^{-}, k}^{\chi, \beta}$ be the left-and right-sided generalized $k$-conformable fractional integrals operators of order $k>0, \mathfrak{R e}(\beta)>0$ and $0<\chi \leq 1$. Let $\psi:[\phi, \varphi] \rightarrow \mathbb{R}$ be a positive mapping with $\psi \in L_{1}[\phi, \varphi]$ and $0 \leq \phi<\varphi$. If $\psi$ is $h$-convex on $[\phi, \varphi]$, then

$$
\begin{aligned}
\psi\left(\frac{\phi+\varphi}{2}\right) & \leq \frac{h\left(\frac{1}{2}\right) \chi^{\frac{\beta}{k}} \Gamma_{k}(\beta+k)}{(\varphi-\phi)^{\chi \beta k}}\left[\mathfrak{J}_{\phi^{+}, k}^{\chi, \beta} \psi(\varphi)+\mathfrak{J}_{\varphi^{-}, k}^{\chi, \beta} \psi(\phi)\right] \\
& \leq \frac{\chi \beta}{k} h\left(\frac{1}{2}\right)[\psi(\phi)+\psi(\varphi)] \int_{0}^{1}\left(1-v^{\chi}\right)^{\frac{\beta}{k}-1} v^{\chi-1}[h(v)+h(1-v)] d v .
\end{aligned}
$$

Proof Since $\psi$ is an $h$-convex function, we can write

$$
\psi(v x+(1-v) y) \leq h(v) \psi(x)+h(1-v) \psi(y)
$$

and

$$
\psi((1-v) x+v y) \leq h(1-v) \psi(x)+h(v) \psi(y) .
$$


Let $v=\frac{1}{2}$, then

$$
\psi\left(\frac{x+y}{2}\right) \leq h\left(\frac{1}{2}\right) \psi(x)+h\left(\frac{1}{2}\right) \psi(y)=h\left(\frac{1}{2}\right)[\psi(x)+\psi(y)]
$$

Assume $x=v \phi+(1-v) \varphi$ and $y=\phi(1-v)+\varphi v$, then we have

$$
\psi\left(\frac{\phi+\varphi}{2}\right) \leq h\left(\frac{1}{2}\right)[\psi(\nu \phi+(1-v) \varphi)+\psi(\phi(1-v)+v \varphi)]
$$

Multiplying the above inequality by $\left(1-v^{\chi}\right)^{\frac{\beta}{k}-1} v^{x-1}$ and then integrating with respect to $v$ over $[0,1]$, we get

$$
\begin{aligned}
\frac{k}{\chi \beta} \psi\left(\frac{\phi+\varphi}{2}\right) \leq & h\left(\frac{1}{2}\right)\left[\int_{0}^{1}\left(1-v^{\chi}\right)^{\frac{\beta}{k}-1} v^{\chi-1} \psi(v \phi+(1-v) \varphi) d v\right. \\
& \left.+\int_{0}^{1}\left(1-v^{\chi}\right)^{\frac{\beta}{k}-1} v^{\chi-1} \psi((1-v) \phi+v \varphi) d v\right] .
\end{aligned}
$$

Now, let $v \phi+(1-v) \varphi=u$ and $(1-v) \phi+v \varphi=v$, then (4.3) becomes

$$
\begin{aligned}
& \frac{k}{\chi \beta} \psi\left(\frac{\phi+\varphi}{2}\right) \\
& \leq \frac{h\left(\frac{1}{2}\right) \chi^{\frac{\beta}{k}-1} \Gamma_{k}(\beta)}{(\varphi-\phi)^{\frac{\chi \beta}{k}}}\left[\frac{\chi^{1-\frac{\beta}{k}}}{\Gamma_{k}(\beta)} \int_{\phi}^{\varphi}\left((\varphi-\phi)^{\chi}-(\varphi-u)^{\chi}\right)^{\frac{\beta}{k}-1}(\varphi-u)^{\chi-1} \psi(u) d u\right. \\
& \left.\quad+\frac{\chi^{1-\frac{\beta}{k}}}{\Gamma_{k}(\beta)} \int_{\phi}^{\varphi}\left((\varphi-\phi)^{\chi}-(v-\phi)^{\chi}\right)^{\frac{\beta}{k}-1}(v-\phi)^{\chi-1} \psi(v) d v\right],
\end{aligned}
$$

which can be written as

$$
\psi\left(\frac{\phi+\varphi}{2}\right) \leq \frac{h\left(\frac{1}{2}\right) \chi^{\frac{\beta}{k}} \Gamma_{k}(\beta+k)}{(\varphi-\phi)^{\frac{\chi \beta}{k}}}\left[\mathfrak{J}_{\phi^{+}, k}^{\chi, \beta} \psi(\varphi)+\mathfrak{J}_{\varphi^{-}, k}^{\chi, \beta} \psi(\phi)\right] .
$$

Now, by replacing $x=\phi, y=\varphi$ in (4.1) and (4.2), respectively, then adding we have

$$
\psi(v \phi+(1-v) \varphi)+\psi((1-v) \phi+v \varphi) \leq[h(v)+h(1-v)][\psi(\phi)+\psi(\varphi)] .
$$

Multiplying (4.5) by $\left(1-v^{\chi}\right)^{\frac{\beta}{k}-1} v^{\chi-1}$ and then integrating over $[0,1]$, we have

$$
\begin{aligned}
& \frac{h\left(\frac{1}{2}\right) \chi^{\frac{\beta}{k}} \Gamma_{k}(\beta+k)}{(\varphi-\phi)^{\frac{\chi \beta}{k}}}\left[\mathfrak{J}_{\phi^{+}, k}^{\chi, \beta} \psi(\varphi)+\mathfrak{J}_{\varphi^{-}, k}^{\chi, \beta} \psi(\phi)\right] \\
& \quad \leq \frac{\chi \beta}{k} h\left(\frac{1}{2}\right)[\psi(\phi)+\psi(\varphi)] \int_{0}^{1}\left(1-v^{\chi}\right)^{\frac{\beta}{k}-1} v^{\chi-1}[h(\nu)+h(1-v)] d v .
\end{aligned}
$$

Now, from (4.4) and (4.6), we get the desired result. 
Theorem 4.2 Let $\psi:[\phi, \varphi] \rightarrow \mathbb{R}$ be a differentiable function on $(\phi, \varphi)$ with $\phi<\varphi$ and $\psi \in L_{1}[\phi, \varphi]$. Then the following equality for $k$-conformable fractional integral holds:

$$
\begin{aligned}
& \frac{\psi(\phi)+\psi(\varphi)}{2}-\frac{\chi^{\frac{\beta}{k}} \Gamma_{k}(\beta+k)}{2(\varphi-\phi)^{\frac{\chi \beta}{k}}}\left[\mathfrak{J}_{\phi^{+}, k}^{\chi, \beta} \psi(\varphi)+\mathfrak{J}_{\varphi^{-}, k}^{\chi, \beta} \psi(\phi)\right] \\
& =\frac{(\varphi-\phi)}{2} \int_{0}^{1}\left[\left(1-\wp^{\chi}\right)^{\frac{\beta}{k}}-\left(1-(1-\wp)^{\chi}\right)^{\frac{\beta}{k}}\right] \psi^{\prime}(\wp \phi+(1-\wp) \varphi) d \wp .
\end{aligned}
$$

Proof Consider

$$
\begin{aligned}
I= & \int_{0}^{1}\left[\left(1-\wp^{\chi}\right)^{\frac{\beta}{k}}-\left(1-(1-\wp)^{\chi}\right)^{\frac{\beta}{k}}\right] \psi^{\prime}(\wp \phi+(1-\wp) \varphi) d \wp \\
= & \int_{0}^{1}\left[\left(1-\wp^{\chi}\right)^{\frac{\beta}{k}}\right] \psi^{\prime}(\wp \phi+(1-\wp) \varphi) d \wp \\
& -\int_{0}^{1}\left[\left(1-(1-\wp)^{\chi}\right)^{\frac{\beta}{k}}\right] \psi^{\prime}(\wp \phi+(1-\wp) \varphi) d \wp \\
= & I_{1}-I_{2} .
\end{aligned}
$$

Integrating by parts and using substitution $u=\wp \phi+(1-\wp) \varphi$, we have

$$
\begin{aligned}
I_{1} & =\int_{0}^{1}\left[\left(1-\wp^{\chi}\right)^{\frac{\beta}{k}}\right] \psi^{\prime}(\wp \phi+(1-\wp) \varphi) d \wp \\
& =\frac{\psi(\varphi)}{\varphi-\phi}-\frac{\beta \chi}{k(\varphi-\phi)} \int_{0}^{1}\left(1-\wp^{\chi}\right)^{\frac{\beta}{k}-1} \wp^{\chi-1} \psi(\wp \phi+(1-\wp) \varphi) d \wp \\
& =\frac{\psi(\varphi)}{\varphi-\phi}-\frac{\beta \chi}{k(\varphi-\phi)^{\frac{\chi \beta}{k}+1}} \int_{\phi}^{\varphi}\left((\varphi-\phi)^{\chi}-(\varphi-u)^{\chi}\right)^{\frac{\beta}{k}-1}(\varphi-u)^{\chi-1} \psi(u) d u \\
& =\frac{\psi(\varphi)}{\varphi-\phi}-\frac{\chi^{\frac{\beta}{k}} \Gamma_{k}(\beta+k)}{(\varphi-\phi)^{\frac{\chi \beta}{k}+1}} \mathfrak{J}_{\varphi^{-}, k}^{\chi, \beta} \psi(\phi) .
\end{aligned}
$$

Also, we have

$$
\begin{aligned}
I_{2} & =\int_{0}^{1}\left[\left(1-(1-\wp)^{\chi}\right)^{\frac{\beta}{k}}\right] \psi^{\prime}(\wp \phi+(1-\wp) \varphi) d \wp \\
& =\frac{-\psi(\phi)}{\varphi-\phi}+\frac{\beta \chi}{k(\varphi-\phi)} \int_{0}^{1}\left(1-(1-\wp)^{\chi}\right)^{\frac{\beta}{k}-1}(1-\wp)^{\chi-1} \psi(\wp \phi+(1-\wp) \varphi) d \wp \\
& =\frac{-\psi(\phi)}{\varphi-\phi}+\frac{\beta \chi}{k(\varphi-\phi)^{\frac{\chi \beta}{k}}+1} \int_{\phi}^{\varphi}\left((\varphi-\phi)^{\chi}-(u-\phi)^{\chi}\right)^{\frac{\beta}{k}-1}(u-\phi)^{\chi-1} \psi(u) d u \\
& =\frac{-\psi(\phi)}{\varphi-\phi}+\frac{\chi^{\frac{\beta}{k}} \Gamma_{k}(\beta+k)}{(\varphi-\phi)^{\frac{\chi \beta}{k}+1}} \mathfrak{J}_{\phi^{+}, k}^{\chi, \beta} \psi(\varphi) .
\end{aligned}
$$

By using the obtained values of $I_{1}$ and $I_{2}$ in (4.7) and then multiplying the result by $\frac{\varphi-\phi}{2}$, we get the desired result.

Theorem 4.3 Let $\psi:[\phi, \varphi] \rightarrow \mathbb{R}$ be a differentiable function on $(\phi, \varphi)$ with $\psi^{\prime} \in L_{1}[\phi, \varphi]$. If $\left|\psi^{\prime}\right|$ is an h-convex function on $[\phi, \varphi]$, then the following inequality for fractional con- 
formable integral holds:

$$
\begin{aligned}
& \left|\frac{\psi(\phi)+\psi(\varphi)}{2}-\frac{\chi^{\frac{\beta}{k}} \Gamma_{k}(\beta+k)}{2(\varphi-\phi)^{\frac{\chi \beta}{k}}}\left[\mathfrak{J}_{\phi^{+}, k}^{\chi, \beta} \psi(\varphi)+\mathfrak{J}_{\varphi^{-}, k}^{\chi, \beta} \psi(\phi)\right]\right| \\
& \leq \frac{\varphi-\phi}{2}\left[\left|\psi^{\prime}(\phi)\right| \int_{0}^{1}\left[\left(1-\wp^{\chi}\right)^{\frac{\beta}{k}}+\left(1-(1-\wp)^{\chi}\right)^{\frac{\beta}{k}}\right] h(\wp) d \wp\right. \\
& \left.\quad+\left|\psi^{\prime}(\varphi)\right| \int_{0}^{1}\left[\left(1-\wp^{\chi}\right)^{\frac{\beta}{k}}+\left(1-(1-\wp)^{\chi}\right)^{\frac{\beta}{k}}\right] h(1-\wp) d \wp\right] .
\end{aligned}
$$

Proof By using Lemma 4.2, modulus property, and $h$-convexity, we have

$$
\begin{aligned}
& \left|\frac{\psi(\phi)+\psi(\varphi)}{2}-\frac{\chi^{\frac{\beta}{k}} \Gamma_{k}(\beta+k)}{2(\varphi-\phi)^{\frac{\chi \beta}{k}}}\left[\mathfrak{J}_{\phi^{+}, k}^{\chi, \beta} \psi(\varphi)+\mathfrak{J}_{\varphi^{-}, k}^{\chi, \beta} \psi(\phi)\right]\right| \\
& =\left|\frac{(\varphi-\phi)}{2} \int_{0}^{1}\left[\left(1-\wp^{\chi}\right)^{\frac{\beta}{k}}-\left(1-(1-\wp)^{\chi}\right)^{\frac{\beta}{k}}\right] \psi^{\prime}(\wp \phi+(1-\wp) \varphi) d \wp\right| \\
& \leq \frac{(\varphi-\phi)}{2}\left|\int_{0}^{1}\left[\left(1-\wp^{\chi}\right)^{\frac{\beta}{k}}+\left(1-(1-\wp)^{\chi}\right)^{\frac{\beta}{k}}\right] \psi^{\prime}(\wp \phi+(1-\wp) \varphi) d \wp\right| \\
& \leq \frac{(\varphi-\phi)}{2} \int_{0}^{1}\left[\left(1-\wp^{\chi}\right)^{\frac{\beta}{k}}+\left(1-(1-\wp)^{\chi}\right)^{\frac{\beta}{k}}\right]\left[h(\wp)\left|\psi^{\prime}(\phi)\right|+h(1-\wp)\left|\psi^{\prime}(\varphi)\right|\right] d \wp \\
& =\frac{(\varphi-\phi)}{2}\left[\left|\psi^{\prime}(\phi)\right| \int_{0}^{1}\left[\left(1-\wp^{\chi}\right)^{\frac{\beta}{k}}+\left(1-(1-\wp)^{\chi}\right)^{\frac{\beta}{k}}\right] h(\wp) d \wp\right. \\
& \left.\quad+\left|\psi^{\prime}(\varphi)\right| \int_{0}^{1}\left[\left(1-\wp^{\chi}\right)^{\frac{\beta}{k}}+\left(1-(1-\wp)^{\chi}\right)^{\frac{\beta}{k}}\right] h(1-\wp) d \wp\right],
\end{aligned}
$$

which completes the proof.

Corollary 4.4 If we take $h(\wp)=1$ in Theorem 4.3, then we get the result presented below:

$$
\begin{gathered}
\left|\frac{\psi(\phi)+\psi(\varphi)}{2}-\frac{\chi^{\frac{\beta}{k}} \Gamma_{k}(\beta+k)}{2(\varphi-\phi)^{\frac{\chi \beta}{k}}}\left[\mathfrak{J}_{\phi^{+}, k}^{\chi, \beta} \psi(\varphi)+\mathfrak{J}_{\varphi^{-}, k}^{\chi, \beta} \psi(\phi)\right]\right| \\
\leq \frac{(\varphi-\phi)}{\chi}\left[\left|\psi^{\prime}(\phi)\right|+\left|\psi^{\prime}(\varphi)\right|\right] \mathbf{B}\left(\frac{1}{\chi}, \frac{\beta}{k}+1\right),
\end{gathered}
$$

where $\mathbf{B}$ denotes the usual beta function.

Corollary 4.5 If we take $h(\wp)=\wp$ in Theorem 4.3 , then we get the result for simple convex function presented below.

$$
\begin{aligned}
& \left|\frac{\psi(\phi)+\psi(\varphi)}{2}-\frac{\chi^{\frac{\beta}{k}} \Gamma_{k}(\beta+k)}{2(\varphi-\phi)^{\frac{\chi \beta}{k}}}\left[\mathfrak{J}_{\phi^{+}, k}^{\chi, \beta} \psi(\varphi)+\mathfrak{J}_{\varphi^{-}, k}^{\chi, \beta} \psi(\phi)\right]\right| \\
& \leq \frac{(\varphi-\phi)}{\chi}\left[\frac{\left|\psi^{\prime}(\phi)\right|+\left|\psi^{\prime}(\varphi)\right|}{2}\right] \mathbf{B}\left(\frac{1}{\chi}, \frac{\beta}{k}+1\right) .
\end{aligned}
$$


Corollary 4.6 If we take $h(\wp)=\wp^{s}$ in Theorem 4.3, where $s \in(0,1)$, then the result for geometric-arithmetically s-convex functions is as follows:

$$
\begin{aligned}
& \left|\frac{\psi(\phi)+\psi(\varphi)}{2}-\frac{\chi^{\frac{\beta}{k}} \Gamma_{k}(\beta+k)}{2(\varphi-\phi)^{\frac{\chi \beta}{k}}}\left[\mathfrak{J}_{\phi^{+}, k}^{\chi, \beta} \psi(\varphi)+\mathfrak{J}_{\varphi^{-}, k}^{\chi, \beta} \psi(\phi)\right]\right| \\
& \leq \\
& \quad \frac{(\varphi-\phi)}{\chi}\left[\frac{\left|\psi^{\prime}(\phi)\right|+\left|\psi^{\prime}(\varphi)\right|}{2}\right]\left[\mathbf{B}\left(\frac{s+1}{\chi}, \frac{\beta}{k}+1\right)\right. \\
& \left.\quad+\mathbf{B}\left(\frac{1}{\chi}, \frac{\beta}{k}+1\right){ }_{2} \mathfrak{F}_{1}\left(-s, \frac{1}{\chi} ; \frac{\chi k+\chi \beta+k}{\chi k} ; u^{\frac{1}{\chi}-1}\right)\right] .
\end{aligned}
$$

\section{Applications to quadrature formulae}

This section consists some particular inequalities which generalize some classical results like the trapezoid inequality. Also Hadamard's inequality can be observed.

Proposition 5.1 (Hadamard's inequality) By using the assumptions of Theorem 2.1 with $\chi=1, s=0$, and $k=1$, we get the following Hadamard's inequality:

$$
\psi\left(\frac{\phi+\varphi}{2}\right) \leq \frac{1}{\varphi-\phi} \int_{\phi}^{\varphi} \psi(\nu) d v \leq \frac{\psi(\phi)+\psi(\varphi)}{2} .
$$

Proposition 5.2 By using the assumptions of Theorem 3.1 with $\chi=1, \beta=1$, and $h(v)=v$, we get the Hadamard's inequality (5.1).

Proposition 5.3 By utilizing the assumptions of Corollary 3.5 with $\chi=1$ and $\beta=1$, we get the following "trapezoid inequality":

$$
\left|(\varphi-\phi) \frac{\psi(\phi)+\psi(\varphi)}{2}-\int_{\phi}^{\varphi} \psi(\nu) d v\right| \leq \frac{(\varphi-\phi)^{2}}{2}\left[\left|\psi^{\prime}(\phi)\right|+\left|\psi^{\prime}(\varphi)\right|\right] .
$$

Proposition 5.4 By utilizing the assumptions of Corollary 3.6 with $\chi=1$ and $\beta=1$, we get the following "trapezoid inequality":

$$
\left|(\varphi-\phi) \frac{\psi(\phi)+\psi(\varphi)}{2}-\int_{\phi}^{\varphi} \psi(v) d v\right| \leq \frac{(\varphi-\phi)^{2}}{6}\left[\left|\psi^{\prime}(\phi)\right|+\left|\psi^{\prime}(\varphi)\right|\right] .
$$

Proposition 5.5 By using the assumptions of Theorem 2.4 with $\chi=1, k=1$, and $s=0$, we get the following "trapezoid inequality":

$$
\left|(\varphi-\phi) \frac{\psi(\phi)+\psi(\varphi)}{2}-\int_{\phi}^{\varphi} \psi(v) d v\right| \leq \frac{(\varphi-\phi)^{2}}{2}\left(\frac{\left|\psi^{\prime}(\phi)\right|^{g}+\left|\psi^{\prime}(\varphi)\right|^{g}}{2}\right)^{\frac{1}{g}} .
$$

\section{Conclusions}

In the current article, we presented generalizations of some mean-type inequalities for fractional integrals of $(k, s)$-Riemann and conformable type. For this purpose, we utilized the $(s+1)$ - and $h$-convex mappings. This work includes equalities so that we can make progress in finding more inequalities by using different functions. The validity of the results is illustrated by considering different convex functions and then by plotting graphs. The presented work includes quadrature formulas as bounds of novel inequalities. The 
findings of this investigation complement those of previous studies. Simply, the recent study confirms the earlier results and plays an additional role by making generalizations.

\author{
Acknowledgements \\ The corresponding author would like to thank Prince Sultan University for funding this work through research group \\ Nonlinear Analysis Methods in Applied Mathematics (NAMAM) group number RG-DES-2017-01-17.
}

Funding

There is no funding for this work.

Availability of data and materials

Not applicable.

Competing interests

The authors declare no potential conflict of interests.

Authors' contributions

All authors jointly worked on the results and they read and approved the final manuscript.

\title{
Author details
}

${ }^{1}$ Department of Mathematics, University of Sargodha, Sargodha, 40100, Pakistan. ${ }^{2}$ Department of Mathematics, University of Management and Technology, Sialkot Campus, Punjab, 51310 Sialkot, Pakistan. ${ }^{3}$ Department of Mathematics and General Sciences, Prince Sultan University, 11586 Riyadh, Saudi Arabia. ${ }^{4}$ Department of Medical Research, China Medical University, 40402 Taichung, Taiwan. ${ }^{5}$ Department of Computer Science and Information Engineering, Asia University, 40402 Taichung, Taiwan. ${ }^{6}$ Department of Mathematics, Shaheed Benazir Bhutto University, Sheringal Upper Dir, Pakistan. ${ }^{7}$ Department of Mathematics, College of Arts and Sciences, Prince Sattam bin Abdulaziz University, 11991 Wadi Aldawaser, Saudi Arabia.

\section{Publisher's Note}

Springer Nature remains neutral with regard to jurisdictional claims in published maps and institutional affiliations.

Received: 19 May 2020 Accepted: 3 August 2020 Published online: 12 August 2020

\section{References}

1. Adjabi, Y., Jarad, F., Abdeljawad, T.: On generalized fractional operators and a Gronwall type inequality with applications. Filomat 31(17), 5457-5473 (2017)

2. Andrews, G.E., Askey, R., Roy, R.: Special Functions. Cambridge University Press, Cambridge (1999)

3. Bainov, D.D., Simeonov, P.S.: Integral Inequalities and Applications, vol. 57. Springer, Berlin (2013)

4. Bandle, C., Gilányi, A., Losonczi, L., Páles, Z., Plum, M.: Inequalities and Applications. Conference on Inequalities and Applications, vol. 157. Birkhäuser, Berlin (2008)

5. Dahmani, Z., Belarbi, S.: On some new fractional integral inequalities. J. Inequal. Pure Appl. Math. 10(3), 86 (2009)

6. Dragomir, S.S.: Hermite-Hadamard's type inequalities for operator convex functions. Appl. Math. Comput. 218, 766-772 (2011)

7. Fernandez, A., Mohammed, P.O.: Hermite-Hadamard inequalities in fractional calculus defined using Mittag-Leffler kernels. Math. Methods Appl. Sci., 1-18 (2020)

8. Habib, S., Mubeen, S., Naeem, M.N.: Chebyshev type integral inequalities for generalized $k$-fractional conformable integrals. J. Inequal. Spec. Funct. 9(4), 53-65 (2018)

9. Iscan, l.: Hermite Hadamard type inequalities for p-convex functions. Int. J. Anal. Appl. 11, 137-145 (2016)

10. Jarad, F., Ugurlu, E., Abdeljawad, T., Baleanu, D.: On a new class of fractional operators. Adv. Differ. Equ. 2017, 247 (2017)

11. Liao, Y., Deng, J., Wang, J.: Riemann-Liouville fractional Hermite-Hadamard inequalities, part II, for twice differentiable geometric-arithmetically s-convex functions. J. Inequal. Appl. 2013, 443 (2013)

12. Mohammed, P.O., Brevik, I.: A new version of the Hermite-Hadamard inequality for Riemann-Liouville fractional integrals. Symmetry 12(610), 1-11 (2020)

13. Mohammed, P.O., Sarikaya, M.Z.: On generalized fractional integral inequalities for twice differentiable convex functions. J. Comput. Appl. Math. 372, 112740 (2020)

14. Niculescu, C.P., Persson, L.E.: In Convex Functions and Their Applications. A Contemporary Approach. CMC Books in Mathematics, New York (2004)

15. Özdemir, M.E., Avci, M., Kavurmaci, H.: Hermite-Hadamard-type inequalities via $(\alpha, m)$-convexity. Comput. Math. Appl. 61(9), 2614-2620 (2011)

16. Özdemir, M.E., Avci, M., Set, E.: On some inequalities of Hermite-Hadamard-type via m-convexity. Appl. Math. Lett. 23(9), 1065-1070 (2010)

17. Rainville, E.D.: Special Functions. Macmillan Co., New York (1960)

18. Rashid, S., Abdeljawad, T., Jarad, F., Noor, M.A.: Some estimates for generalized Riemann-Liouville fractional integrals of exponentially convex functions and their applications. Mathematics 7, 807 (2019)

19. Rashid, S., Akdemir, A.O., Jarad, F., Noor, M.A., Noor, K.I.: Simpson's type integral inequalities for k-fractional integrals and their application. AIMS Math. 4(4), 1087-1100 (2019)

20. Rashid, S., Jarad, F., Noor, M.A., Kalsoom, H., Chu, Y.M.: Inequalities by means of generalized proportional fractional integral operators with respect to another function. Mathematics 7(12), 1-18 (2019) 
21. Rashid, S., Jarad, F., Noor, M.A., Noor, K.I., Baleanu, D., Liu, J.B.: On Grüss inequalities within generalized $k$-fractional integrals. Adv. Differ. Equ. 2020, 203 (2020)

22. Samraiz, M., Perveen, Z., Rahman, G., Nisar, K.S., Kumar, D.: On (k, s)-Hilfer-Prabhakar Fractional Derivative with. Applic. Math. Phys. Accepted

23. Sarikaya, M.Z., Aktan, N.: On the generalizations of some integral inequalities and their applications. Math. Comput. Model. 54(9-10), 2175-2182 (2011)

24. Sarikaya, M.Z., Dahmani, Z., Kiriss, M.E., Ahmad, F.: (k, s)-Riemann-Liouville fractional integral and applications. J. Math. Stat. 45(1), 77-89 (2016)

25. Sarikaya, M.Z., Hüseyin, Y:: On Hermite-Hadamard type inequalities for Riemann-Liouville fractional integrals. Miskolc Math. Notes 17, 1049-1059 (2017)

26. Set, E., Akdemir, A.O., Gözpinar, A., Jarad, F.: Ostrowski type inequalities via new fractional conformable integrals. AlMS Math. 4(6), 1684-1697 (2019)

27. Set, E., Akdemir, A.O., Mumcu, l.: The Hermite-Hadamard's inequality and its extensions for conformable fractional integrals of any order $\alpha>0$. Creative Math. Inform. 27(2), 197-206 (2018)

28. Set, E., Özdemir, M.E., Dragomir, S.S.: On the Hadamard-type inequalities involving several kinds of convexity. J. Inequal. Appl. 2010, 286845 (2010)

29. Set, E., Özdemir, M.E., Dragomir, S.S.: On the Hermite-Hadamard inequality and other integral inequalities involving two functions. J. Inequal. Appl. 2010, 148102 (2010)

30. Set, E.J., Gözpnar, A., But, S.I.: A study on Hermite-Hadamard-type inequalities via new fractional conformable integrals. Asian-Eur. J. Math. 2021, 2150016 (2021)

31. Varosanec, S.: On h-convexity. J. Math. Anal. Appl. 326, 303-311 (2007)

32. Wu, X., Wang, J., Zhang, J.: Hermite Hadamard-type inequalities for convex functions via the fractional integrals with exponential kernel. Mathematics 7, 845 (2019)

33. Zhang, Y., Wang, J.: On some new Hermite-Hadamard inequalities involving Riemann-Liouville fractional integrals. J. Inequal. Appl. 2013, 220 (2013)

\section{Submit your manuscript to a SpringerOpen ${ }^{\circ}$ journal and benefit from:}

- Convenient online submission

- Rigorous peer review

- Open access: articles freely available online

- High visibility within the field

- Retaining the copyright to your article

Submit your next manuscript at $\gg$ springeropen.com 